Conclusion A large proportion of our referrals are to investigate Crohn's disease. Most have had multiple endoscopic and imaging modalities prior to referral. DBE is more likely to have positive findings when associated with abnormal imaging rather than abnormal VCE findings. Only those with abnormalities on imaging other than VCE were confirmed to have Crohn's disease; it may be that subtle inflammatory changes on VCE are over reported or that findings were beyond the reach of DBE. Our figures although small would suggest that in those with normal radiological imaging there is little improvement in diagnostic yield with DBE.

Competing interests None declared.

\section{PMO-211 PROPOFOL SEDATION FOR COLONOSCOPY: A SINGLE CENTRE'S EXPERIENCE}

doi:10.1136/gutjnl-2012-302514b.211

D Rogers, ${ }^{*}$ R Robinson, S Shah. University Hospitals Leicester, Leicester, UK

Introduction GI endoscopy has been widely practiced for nearly 40 years. Techniques and sedation regimes have advanced together with an ever increasing complexity of therapeutic possibilities. Despite improved colonoscopic technique there remain a small number of patients who cannot tolerate colonoscopy. We introduced propofol endoscopy lists for difficult patients and complex therapeutic work. The lists are run by an anaesthetist and aim to ensure that the most technically challenging patients are comfortable, relaxed and compliant during the procedure. We review the success and complications of colonoscopy under propofol in our centre.

Methods Review of the last 100 consecutive colonoscopies performed under propofol at Leicester General Hospital. Data were analysed for demographics, indications, diagnoses, propofol dose, reason for the use of propofol and complications. Polyp detection figures were compared to JAG standards and we assessed completion rates in those who had had a failed procedure under conscious sedation previously.

Results 100 procedures were analysed and the patients' age ranged from 20 to 84 years with $70 \%$ female and $30 \%$ male. Mean propofol dose was $328 \mathrm{mg}$. 66 patients had had a previous colonoscopy of which $50 \%$ had been failed. In the other $50 \%$ a variety of reasons were given for propofol use. Of the 34 patients who had not undergone previous colonoscopy the reason for using propofol was only clear in 9 . Polyps were detected in $29 \%$ of procedures and $89 \%$ were completed successfully. $85 \%$ of procedures in those patients who had previously failed colonoscopy under sedation were successful. Poor bowel prep prevented completion in three cases, and therefore if these are excluded $93 \%$ of colonoscopies previously failed under conscious sedation were successful with propofol. One procedure that had been successful, but difficult, using conscious sedation was unsuccessful using propofol. This may relate to difficulties turning anaesthetised patients. One patient died within 30 day of their procedure. They had extensive ischaemic colitis and significant cardiac comorbidities.

Conclusion There has been a sustained demand for propofol sedation within UHL, and it appears to be well-tolerated and safe in appropriately selected patients. High risk patients should be identified and directed to more appropriate diagnostic modalities. It is important to remember that propofol is not a panacea, and we describe a procedure that had been "tricky" using conscious sedation becoming impossible when performed under propofol. Propofol has a role to play in complex therapeutic work and in those who cannot otherwise tolerate the procedure due to pain. Propofol lists are popular with patients, and as complex therapeutic endoscopy expands it is likely that all hospitals will need a similar service, but an appropriately negotiated tariff is necessary to take account of increased costs.

Competing interests None declared.
PM0-212 PATIENTS WITH POSITIVE FAECAL OCCULT BLOOD TEST (FOBT) FOLLOWING PREVIOUS LOW RISK COLONOSCOPY IN THE BOWEL CANCER SCREENING PROGRAMME: SHOULD CURRENT APPROACH BE CHANGED?

doi:10.1136/gutjnl-2012-302514b.212

D Nylander, ${ }^{*}$ M Ritchie. Gastroenterology, South of Tyne Bowel Cancer Screening Centre, Tyne and Wear, UK

Introduction In the UK FOBt Bowel Cancer Screening Programme (BCSP), patients between 60 and 75 are invited to submit stool specimens 2 yearly. Those who have either two weakly positive (+ve) or one abnormal FOBt are recommended to undergo colonoscopy. This recommendation stands, even if they have had a previous colonoscopy within the BCSP, regardless of the findings or time frame. In theory therefore a patient may be recommended colonoscopy every 2 years if they have any persisting non-neoplastic lesions that cause bleeding. BSG guidance however recommends surveillance colonoscopy in 5 years (or not at all) for patients with low risk adenoma. ${ }^{1}$ All endoscopists in BCSP have been assessed and deemed competent colonoscopists. Also all procedures are carefully monitored by specialist practitioners. Thus this is the most quality assured setting for colonoscopy practice in the health service. We aimed (a) To determine if there were patients who returned to for 2nd BCSP colonoscopy in under 5 years, despite previous colonoscopy being classed as low risk or non-neoplastic. (b) To determine if 2 nd colonoscopy gave prognostically significant result.

Methods The BCSP database was used to identify cases with 1st colonoscopy reported as normal, low risk adenomas or "abnormal but no adenoma". Any of these who had a 2nd colonoscopy within the BCSP for +ve FOBt were noted and their reports obtained to get the findings of both procedures. The study period was April 2007 to October 2011.

Results 40 patients, deemed low risk at 1st BCSP colonoscopy returned new positive FOBt kits in following screening round. Of these two declined 2nd colonoscopy when contacted (initial colonoscopy findings were one Crohns, one diverticulosis). In three cases, interval between colonoscopies was 4 years, all the rest being 2 years. All colonoscopy findings are in Abstract PMO-212 table 1. All adenomas were $3 \mathrm{~mm}$ or less.

\section{Abstract PM0-212 Table 1}

\begin{tabular}{ll}
\hline $\begin{array}{l}\text { Initial colonoscopy principal } \\
\text { finding (number) }\end{array}$ & $\mathbf{2}^{\text {nd }}$ colonoscopy principal finding (number) \\
\hline Normal (11) & Normal (7), 1 or 2 small adenomas (4) \\
1 or 2 small adenomas (13) & 1 or 2 small adenomas (6), normal (5) \\
Colitis (1) or Crohns (3) & Colitis (1), Crohns $(2 ; 1$ declined) \\
Diverticular disease (9) & Diverticular disease (7), normal (1) \\
Radiation proctitis & Radiation proctitis \\
Angiodysplasia & Normal \\
\hline
\end{tabular}

Conclusion A small number of patients will have positive FOBt tests despite a "low risk" colonoscopy in BCSP for neoplasia within previous 2 years. In our patient group, a 2 nd colonoscopy in this period showed no new prognostically significant findings. Our data suggests that there is no need to deviate from the BSG recommendation and perform repeat procedures for "low risk" patients in $<5$ years in the BCSP.

Competing interests None declared.

\section{REFERENCE}

1. Gut 2010;59:666. 